\title{
Topographical transcriptome mapping of the mouse medial ganglionic eminence by spatially resolved RNA-seq
}

\author{
Sabrina Zechel ${ }^{1}$, Pawel Zajac ${ }^{2}$, Peter Lönnerberg ${ }^{2}$, Carlos F Ibáñez ${ }^{1,3^{*}}$ and Sten Linnarsson ${ }^{2^{*}}$
}

\begin{abstract}
Background: Cortical interneurons originating from the medial ganglionic eminence, MGE, are among the most diverse cells within the CNS. Different pools of proliferating progenitor cells are thought to exist in the ventricular zone of the MGE, but whether the underlying subventricular and mantle regions of the MGE are spatially patterned has not yet been addressed. Here, we combined laser-capture microdissection and multiplex RNA-sequencing to map the transcriptome of MGE cells at a spatial resolution of $50 \mu \mathrm{m}$.

Results: Distinct groups of progenitor cells showing different stages of interneuron maturation are identified and topographically mapped based on their genome-wide transcriptional pattern. Although proliferating potential decreased rather abruptly outside the ventricular zone, a ventro-lateral gradient of increasing migratory capacity was identified, revealing heterogeneous cell populations within this neurogenic structure.

Conclusions: We demonstrate that spatially resolved RNA-seq is ideally suited for high resolution topographical mapping of genome-wide gene expression in heterogeneous anatomical structures such as the mammalian central nervous system.
\end{abstract}

\section{Background}

No other organ in the body harbors the enormous cell diversity that is found in the mammalian brain. Within the telencephalon, cell diversity among inhibitory interneurons vastly exceeds that of excitatory projection neurons. Over 70 different classes of inhibitory interneurons differing in location, morphological, neurochemical and electrophysiological properties are thought to exist in the mammalian cerebral cortex [1,2]. Unlike excitatory neurons, inhibitory (for example, GABAergic) interneurons originate outside the cortex, in transient neurogenic structures of the ventral telencephalon known as the ganglionic eminences. The medial ganglionic eminence (MGE) contributes the majority of cortical interneurons, mainly basket and chandelier cells expressing distinct combinations of neuropeptides, calcium-binding proteins and ion channels [3-5]. How such vast cellular diversity is

\footnotetext{
* Correspondence: carlos.ibanez@ki.se; sten.linnarsson@ki.se

'Department of Neuroscience, Karolinska Institute, Stockholm SE-171 77, Sweden

${ }^{2}$ Department of Medical Biochemistry and Biophysics, Karolinska Institute, Stockholm SE-171 65, Sweden

Full list of author information is available at the end of the article
}

generated, and the degree to which it may be predetermined among progenitors of the ganglionic eminences or acquired en route to the cortex, remain outstanding questions in the field. Previous studies have subdivided the proliferative ventricular zone of the MGE based on the expression pattern of transcription factors known to be involved in forebrain development [4-7]. However, this is expected to account for only a small fraction of the diversity of cortical interneurons, as several postmitotic selector genes and extracellular signals are known to contribute to the differentiation of GABAergic neuron precursors as they progress into the subventricular and mantle zones of the MGE. The gene expression profiles of postmitotic GABAergic progenitors, and whether such profiles may be spatially organized within the MGE, have not yet been investigated.

Spatially resolved gene and protein expression analysis can be achieved by a variety of affinity-based staining methods, such as in situ hybridization and immunofluorescence. However, these methods are limited by the ability of current microscopes to accurately resolve mutltiple fluorophore wavelengths, so that typically less than five genes or 
proteins can be simultaneously detected. In order to increase transcriptome coverage, it is possible to stain adjacent sections, or use multiple animals, one example of which is the very powerful Allen Brain Atlas [8]. However, this approach necessarily limits the resolving power since tissue sections from different animals cannot easily be aligned. Recently, a multiplexed in situ sequencing technique was developed [9], which was capable of simultaneous detection of several tens of genes in tissue sections, with near-single-cell resolution. However, in order to characterize unknown cellular states, it would be desirable to measure the entire transcriptome across a tissue section with single-cell resolution.

We have previously developed a method, termed single-cell tagged reverse transcription (STRT), that enables the characterization of single-cell transcriptional landscapes by highly multiplexed RNA-sequencing (RNA-seq) $[10,11]$. As an initial step towards genomewide transcriptome imaging of tissue sections, we have adapted the STRT method to laser microdissected tissue samples. By systematically sampling the tissue in a regular grid, we isolated $50 \times 50 \times 50 \mu^{3}$ cubes that are akin to the 'voxels' in a three-dimensional volumetric space. Sampling such voxels from a single tissue section and subjecting each voxel to single-cell RNA-seq yields a two-dimensional image where each individual voxel comprises an entire transcriptome dataset. As a result, it is possible to project the expression of any gene onto this two-dimensional image, enabling the equivalent to a whole-genome in situ hybridization. Moreover, clustering voxels based on their expression profiles allows the identification of spatial regions of distinct gene expression patterns, thereby revealing the functional architecture of the tissue. This approach is ideally suited for high resolution topographical mapping of genome-wide gene expression in heterogeneous anatomical structures such as the mammalian central nervous system. Here, we present a proof-of-concept study of this method applied to the mouse MGE. Our analysis revealed topographically distinct groups of progenitor cells showing different stages of interneuron maturation within this neurogenic structure.

\section{Results}

\section{Genome-wide transcriptional imaging of the mouse medial ganglionic eminence}

Our goal was to obtain an unbiased, spatially resolved transcriptome map of the mouse MGE. We reasoned that clustering these primary data would reveal transcriptionally defined subregions corresponding to functionally distinct areas. Fifty-micrometer-thick cryo-sections of the embryonic day (E)12.5 mouse MGE were used to collect $50 \times 50 \times 50 \mu^{3}$ samples (henceforth called 'voxels') by laser microdissection based on a regular grid of compartments, each containing approximately 100 cells, covering the entire MGE (Figure 1A-C). We used STRT $[10,11]$ to generate RNA-seq data, treating each voxel as equivalent to a single cell. Two sections were collected from two wild-type embryos, respectively. A third section was taken from a Gfra $1^{\text {tlz/tlz }}$ mutant embryo [12] as an internal control of the method. We have previously shown that Gfra1 (a receptor for glial cell line-derived neurotrophic factor or GDNF) is expressed in a discrete domain in the ventro-medial MGE [13,14]. In total, 312 samples were analyzed (130 and 94 from the wild-type animals and 94 from the Gfra1 ${ }^{\text {tlz/tlz }}$ mutant), containing 13,884 expressed genes (2,236 genes detected per sample on average). A full transcriptome was associated with each $50 \times 50 \times 50 \mu^{3}$ voxel of the MGE.

Since the tissue was collected in a regular grid, each sample could be represented as a voxel in a two-dimensional image. And since we had obtained the complete transcriptome of each voxel, we could then visualize the expression of any gene in the MGE with $50 \mu \mathrm{m}$ resolution. For example, Figure 1D shows expression heatmaps of Sox 2 and Couptf1 (expressed in dividing cells), Dclk2, Sox11 and Maged1 (expressed by immature interneurons), as well as Nnat, Malat1 and Gap43 (expressed by migrating interneurons), demonstrating that clear spatial patterns can be obtained by this process. The observed patterns were reproducible across animals (Additional file 1). In agreement with previous observations, Gfral expression was localized to the ventromedial portion of the MGE of wild-type embryos, but was not detected in the Gfra $1^{\text {tlz/tlz }}$ embryo [13].

Next, we sought to delineate transcriptionally defined subregions of the MGE; these would be strong candidates for functionally distinct subdivisions containing cells of different types or in different stages of maturation. Clustering is the standard method of classifying and visualizing gene expression datasets [15] and there are many established clustering algorithms such as hierarchical clustering, k-means and affinity propagation. Clustering is often combined with dimension reduction as, for example, in principal component analysis or multidimensional scaling. However, data from highly amplified samples, such as single cells and laser microdissected tissues, are typically noisier and less sensitive than what is normally obtained from bulk RNA samples. We therefore sought to use a clustering method that would be less affected by those aspects of the data. Topological data analysis (TDA) [16] is a recently developed clustering and visualization technique that focuses on the topology of the data in a high-dimensional gene expression space. In TDA, a space of gene expression is defined by the two first principal components. Samples are then grouped by proximity in this space, and merged. Finally, merged groups of samples are clustered based on their pairwise correlation coefficients (see [16] for details). The result is a 


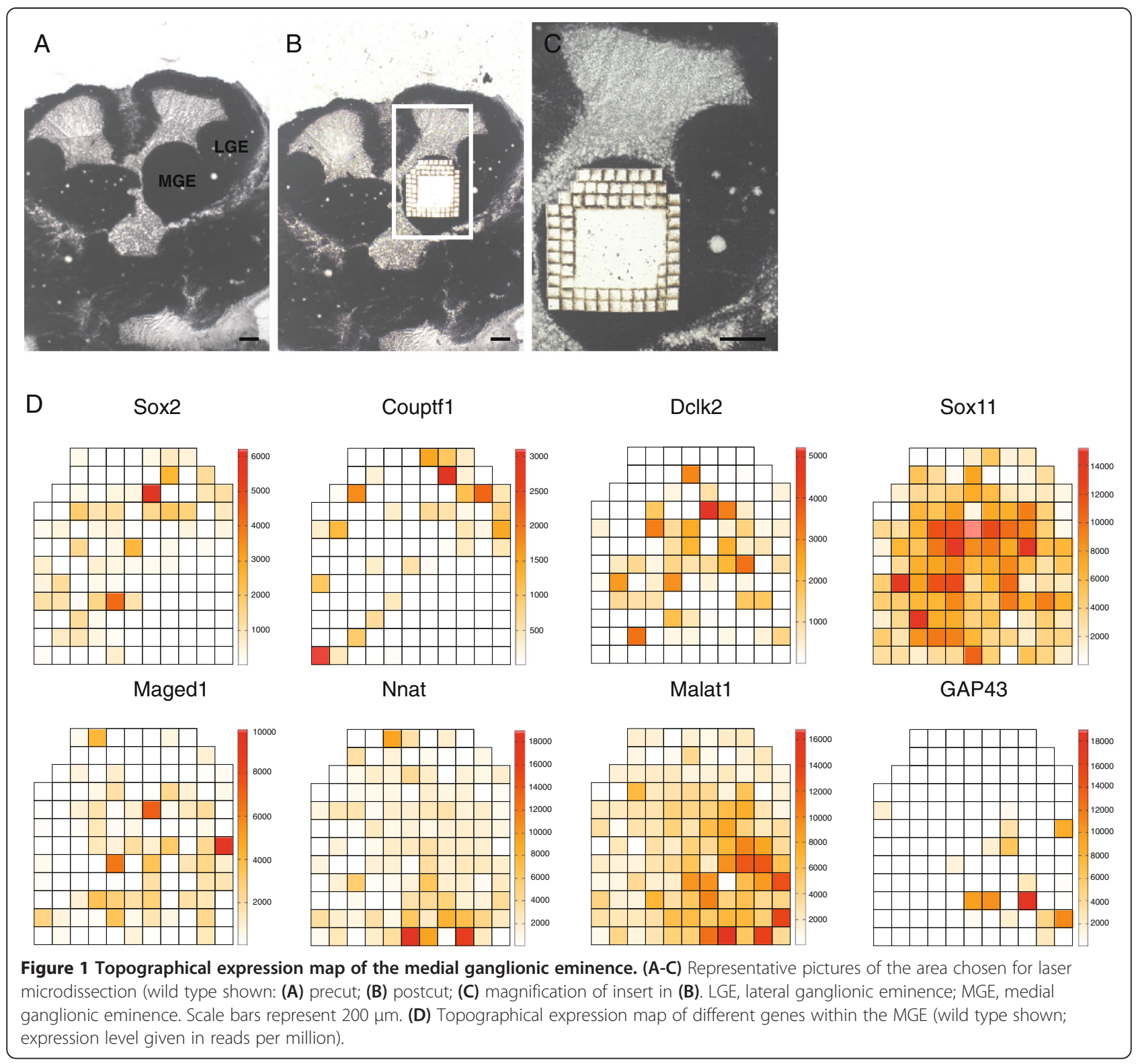

graph linking groups of samples that share gene expression patterns. The graph captures similarity on multiple levels: groups of near-identical samples, linked to distinct but similar samples, and disconnected from samples that show little or no similarity.

We applied TDA on the combined voxels from all three mice. In this way, we could ask whether voxels that were clustered together in one mouse would also cluster with homologous voxels from the other mice. The shape of the TDA graph suggested a one-dimensional progression (Figure 2A). In order to determine if the TDA graph corresponded to spatially defined regions, we segmented the graph into five clusters and projected these back onto the tissue slices (Figure 2B-D). In each mouse, the clusters mapped onto four spatially distinct regions of the MGE, corresponding to a ventrolateral progression from the ventricular zone to the mantle zone. Importantly, voxels belonging to the same cluster mapped to spatially homologous regions in the MGE of the three mice, thus crossvalidating the results. All three mice, including the Gfra $1^{\text {tlz/tlz }}$ mutant, displayed a similar patterning of the MGE (Figure 2B-D), indicating that lack of Gfral does not affect the overall spatial organization of the MGE. Apart from the absence of Gfra1, the only other significant difference in the mutant was a large enrichment in Xist, indicating that this embryo was female. The data from the three mice were therefore pooled for further analysis. Voxels belonging to cluster 1 mapped to the ventricular zone in all three mice (red in Figure 2B-D), suggesting that this cluster corresponds to proliferative cells. Cluster 
A

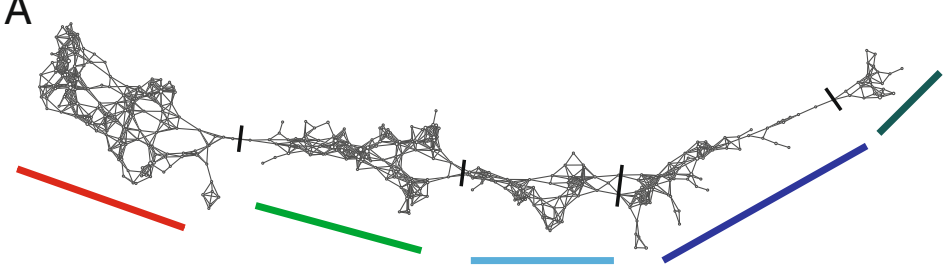

B

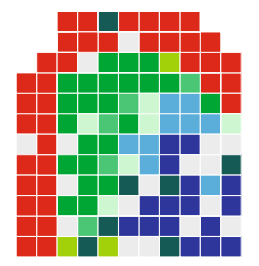

C

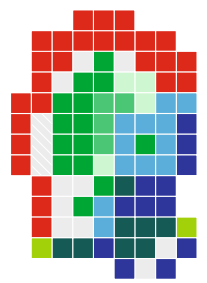

D

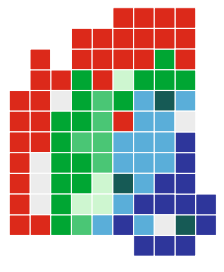

Figure 2 Cluster analysis. (A) Gene expression clusters obtained by Ayasdi analysis (each node representing one voxel). (B-D) Scheme showing the assignment of gene clusters to the MGE in all three animals (wild type (B), wild type (C), Gfra ${ }^{\text {tz/tlz }}$ (D)) used in this study.

2 was localized directly underneath cluster 1 , indicating that cells leaving the ventricular zone display sufficiently large gene expression changes to be detected by our method (green in Figure 2B-D). Cluster 3 was situated further ventrolaterally, clearly demarcated from cluster 2 (light blue in Figure 2B-D). Although distinct at the gene expression level, clusters 4 and 5 did not segregate spatially, both mapping to the most ventrolateral region of the MGE mantle zone, where the most mature progenitors are thought to be located (dark blue in Figure 2B-D). In what follows, these two clusters will be referred to as cluster 4/5.

Next, we sought to determine whether the identified clusters corresponded to distinct biological functions. We performed comparisons of each cluster individually against pooled data from the other clusters and identified genes that best differentiate each cluster from the rest (Kolmogorov-Smirnov test with Bonferroni correction and $\alpha<0.05)$. A selection of these genes is highlighted in Figure 3. Gene Ontology (GO) analysis (DAVID $[17,18]$ ) of the genes expressed by each cluster provided initial insights into the functional properties of the different MGE subdomains. Significant GO terms are indicated in Figure 3. Cluster 1 was defined by genes primarily associated with proliferation and neurogenesis, including genes involved in chromosome and DNA packing (for example, $M t f h f d 1$ ), replication (for example, Mybl2, Pcna) and cell cycle regulation (for example, Ccna2, Cdc73). This cluster was also characterized by the expression of transcription factor E2f1 and cyclin D1 (Ccnd1), with well established functions in the control of cell cycle [19-21]. Cluster 2 was defined by GO terms related to interneuron differentiation, cell morphogenesis and forebrain development, suggesting that this cluster contains interneuron precursors that have left the cell cycle and become postmitotic. These precursor cells turn on expression of selector and terminal differentiation genes that allow fate specific functions, including homeobox transcription factors such as aristaless homeobox gene (Arx), LIM/homeobox protein 6 (Lhx6) and drosophila distalless gene 1 and 5 (Dlx1/5), all known to be crucial for GABAergic interneuron differentiation [22-26]. Cluster 3 was characterized by genes associated with neuron differentiation, but also incorporated genes controlling migration and neuronal projection, including plexinA2 (Plxna2) and kinesin family member 5A (Kif5a). Finally, the GO terms that defined cluster $4 / 5$ were strongly linked to neuronal migration and cell projection, and included many genes encoding extracellular molecules known to regulate these processes, such as semaphorins, neuregulins, neurotrophic factors and receptors (for example, Tgfb2 and Gfra1), chemokines and enzymes for neurotransmitter synthesis (for example, GABA, glutamate).

\section{Spatial pattern validation of medial ganglionic eminence transcriptome clusters}

Validation of the spatial pattern of MGE transcriptome clusters was performed by in situ hybridization for a selected subset of moderately to highly expressed genes from every cluster. In each case, in situ hybridization was combined with immunohistochemistry for Gap43, which defines cluster 4/5 (Figure 4). Sox 2 [27-29] and COUP transcription factor 1 (Couptf1), also known as Nr2f2, [26,30] were chosen as markers for cluster 1, and in situ hybridization for both genes showed their expression restricted to the ventricular zone, as expected (Figure 4A-H). For cluster 2, in situ hybridization for Doublecortin like kinase 2 (Dclk2) and Sox11 [31,32] 


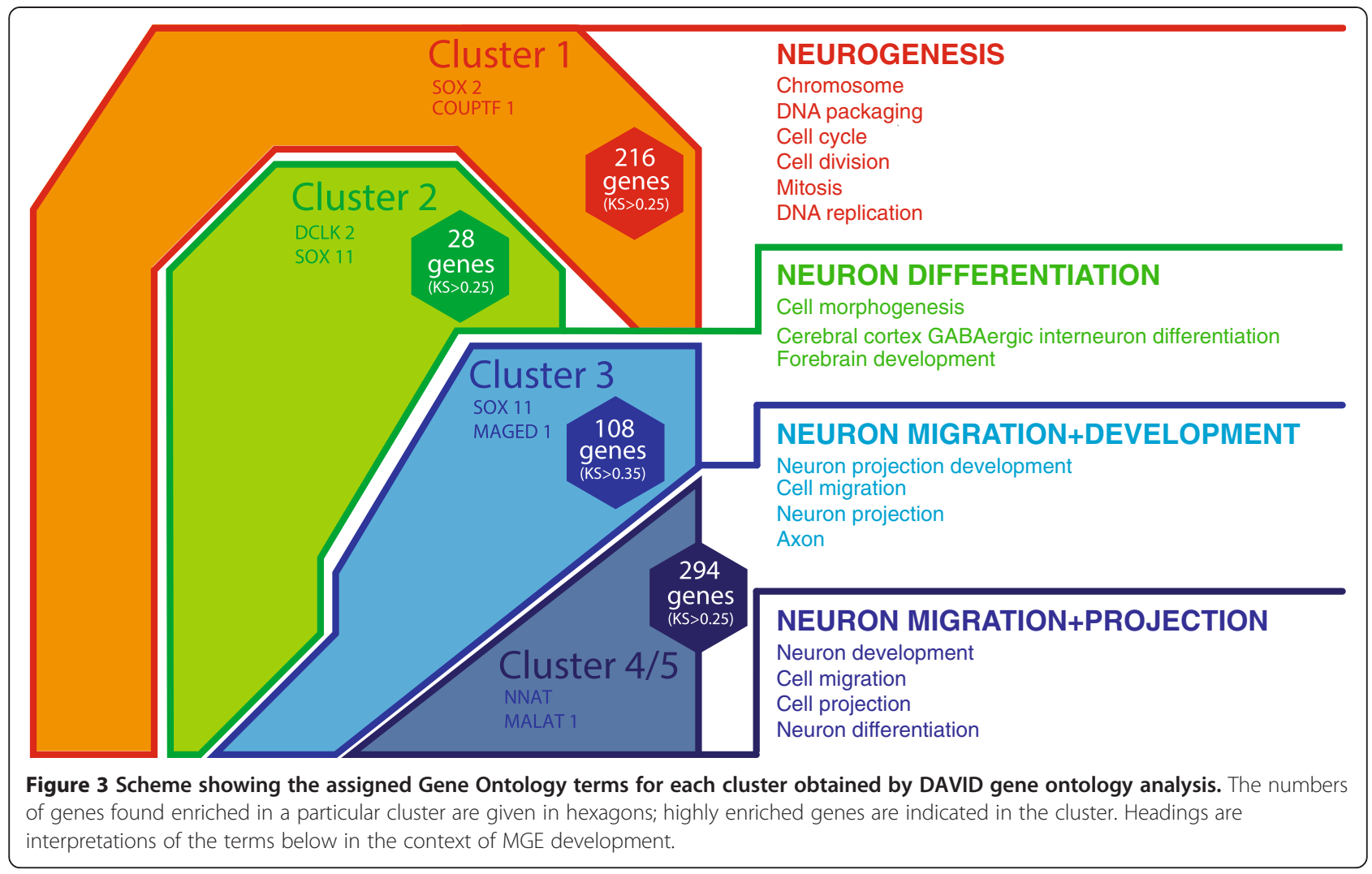

delineated a narrow region immediately ventral to the ventricular zone, matching the expected location of this cluster (Figure 4I-L). In situ hybridization for cluster 3 gene Melanoma antigen family D1 (Maged1) labeled an area immediately dorsal to the Gap43 signal, also matching the expected spatial location of this cluster (Figure 4Q-T). Unlike clusters 1 and 2, cluster 3 genes included transcripts encoding products that regulate cell migration. For example, Maged 1 has been shown to control Dlx-dependent migration-related transcription [33]. We also found that some genes previously linked to GABAergic interneuron differentiation, such as Sox11, were expressed by cells in both cluster 2 and 3 (Figure 4M-P). Cells in the lateral margin of the MGE expressed genes belonging to cluster $4 / 5$, including the cytoskeleton regulator Gap43 [34] and the transmembrane protein gene neuronatin (Nnat) (Figure 4U-X), both of which have previously been linked to cell migration $[34,35]$. The expression of several genes unique to cluster $4 / 5$ is maintained in migrating interneurons as they propagate towards the cortex. One of the genes expressed in both cluster 3 and 4/5, Metastasis associated lung adenocarcinoma transcript 1 (Malat1; Figures 1D and 4Y-B') expresses a non-coding RNA that has been previously shown to regulate proliferation and apoptosis, while also affecting cell migration, a putative distinctive function of cells in cluster $4 / 5$ [36].
Functional properties of topographically mapped medial ganglionic eminence transcriptome clusters

Finally, we wished to validate some of the functions of the different MGE subcompartments predicted by transcriptome imaging, focusing on proliferation and migration. The proliferative activity of each cluster was assessed by incorporation of the thymidine analog bromodeoxyuridine (BrdU) during the $\mathrm{S}$ phase of the cell cycle. As expected, cluster 1, which topographically corresponded to the ventricular zone, contained the majority of proliferative cells ( $87 \%$ of all labeled MGE cells; Figure 5A-D,I). A few scattered cells still undergoing mitotic cell division were also found in cluster 2 (about $17 \%$ of all labeled MGE cells; Figure 5E-H,I), which may correspond to a small subpopulation of MGE transit amplifying cells, as previously noted [37-39]. Clusters 3 and 4/5 were virtually devoid of replicating cells (Figure 5I). In order to assess migratory activities, MGE subcompartments were manually microdissected under a microscope and used to establish explant cultures. Migration was scored as the number of cells that had migrated outside of the explant relative to explant area after $24 \mathrm{~h}$ in culture. The affiliation of each individual explant with a specific cluster was validated retrospectively by in situ hybridization for characteristic clusterspecific genes (Sox2 for cluster 1, Dclk2 for cluster 2, Maged 1 for cluster 3 and Nnat for cluster 4/5) in 


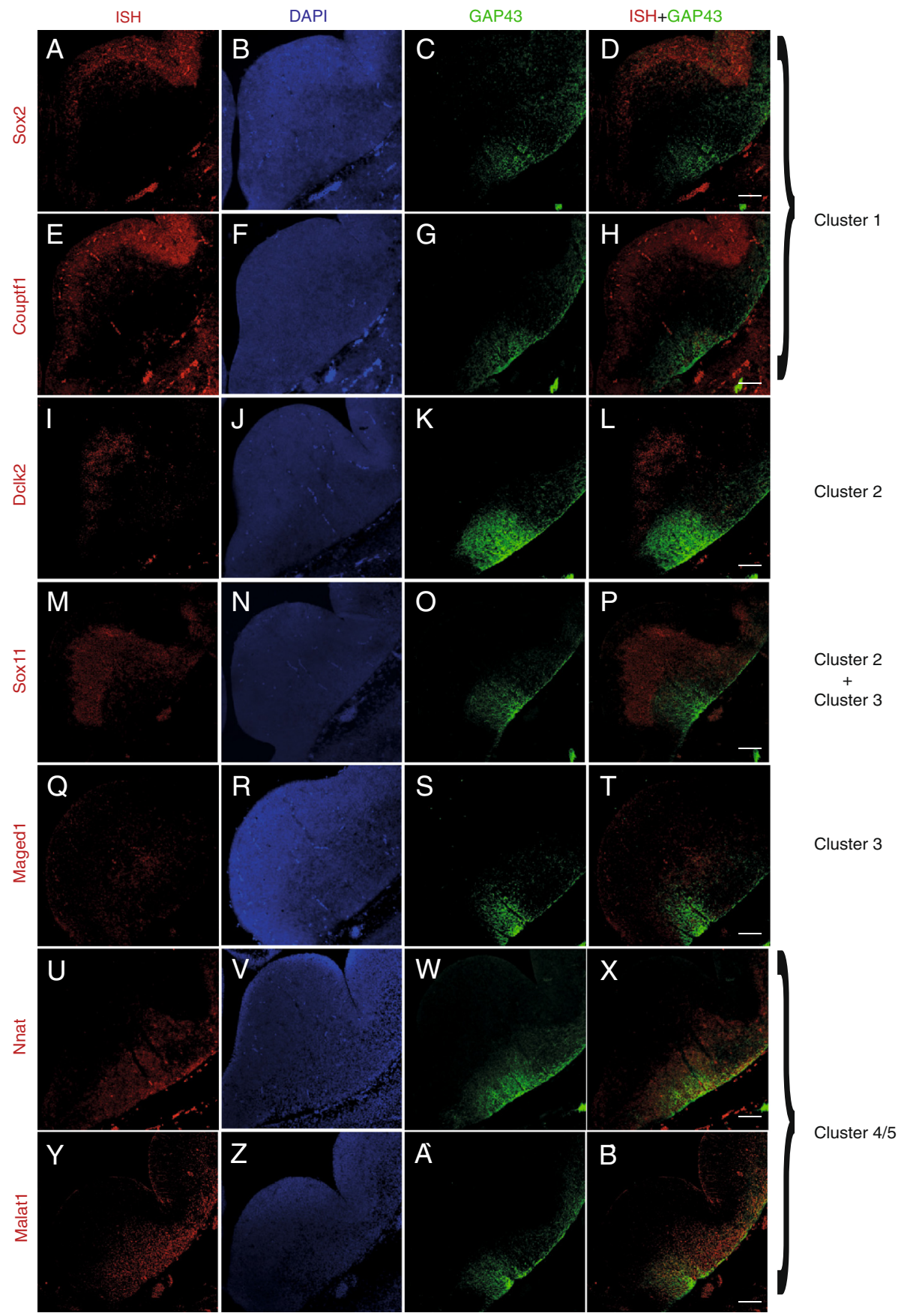

Figure 4 Validation of spatial expression patterns. Validation of genes found by RNA sequencing using in situ hybridization (first column) combined with immunohistochemistry against Gap43 (third column; overlap of both in column 4). Column 2 shows nuclear staining using DAPI (scale bars represent 100 mm). (A-D) Sox2. (E-H) Couptf1. (I-L) Dclk2. (M-P) Sox11. (Q-T) Maged1. (U-X) Nnat. (Y-B') Malat1.

combination with BrdU and Gap43 staining (Additional file 2). Explants expressing markers from more than one cluster were excluded from the analysis. Explants deriving from cluster 1 did not contain migratory active cells (Figure 6A,E). Very few cells were seen leaving explants derived from cluster 2 (Figure 6B,E). Migratory activity increased progressively among cells derived from clusters 3 and 4/5 (Figure 6C-E), in accordance with their prominent expression of genes involved in cytoskeleton remodeling and GABAergic interneuron migration. In summary, while proliferative potential disappeared rather abruptly beyond the ventricular zone, migratory 


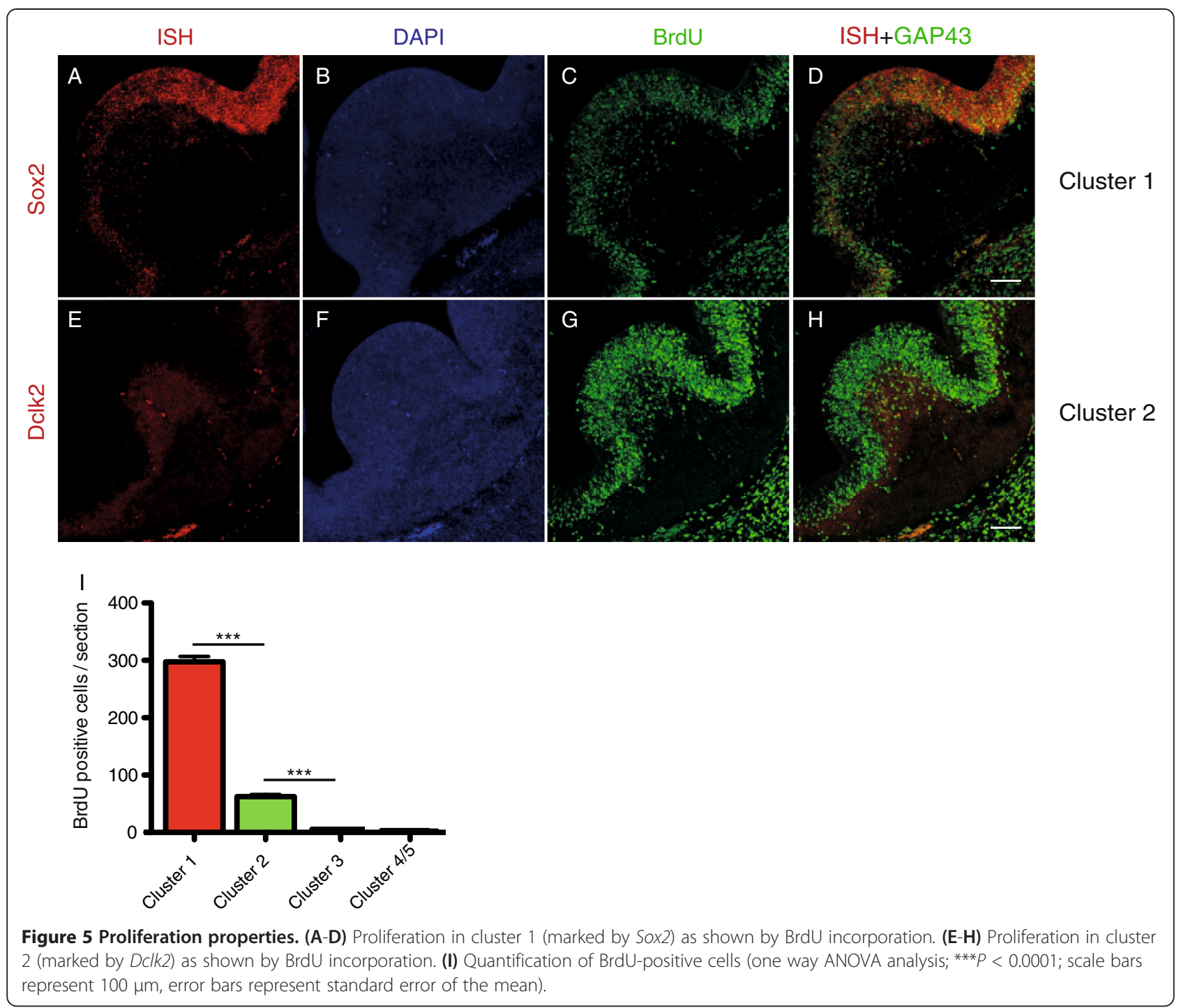

activity appeared to be gradually acquired among postmitotic GABAergic precursors as they reached the most ventrolateral region of the MGE.

\section{Discussion}

Understanding the development of an organ as complex as the mammalian brain requires tools with adequate resolving power and multiplexing capability. The nervous system consists of varied cell types intermingled in complex patterns, whose morphology and position can change on a time scale of minutes to hours. The development and differentiation of mature cell types involves a complex molecular machinery, including RNA, proteins and signaling molecules. Thus, ideally, we seek methods to monitor the dynamics of these molecules with a spatial resolution of a few micrometers and a temporal resolution of seconds to minutes. Moreover, mammalian cell types are characterized by the combinatorial expression of genes and proteins, and the ideal measurement must therefore simultaneously probe all molecules of interest. Unfortunately, the goals of spatial/temporal resolution and whole-genome multiplexing are currently mutually incompatible. RNA can be detected with submicrometer resolution [40], but only in fixed tissues and with very limited multiplexing ability. Proteins can be detected at submicrometer resolution $[41,42]$, even dynamically in living tissues, but again multiplexing is limited to a handful of proteins. Single-cell RNA-seq [10,43] allows whole-transcriptome analysis of single cells. However, these methods require isolated cells, which are typically obtained by dissociation of the target tissue, thus erasing the spatial context.

There is currently a choice between whole-genome methods without spatial information, and spatially resolved methods that target only a few genes or proteins. 

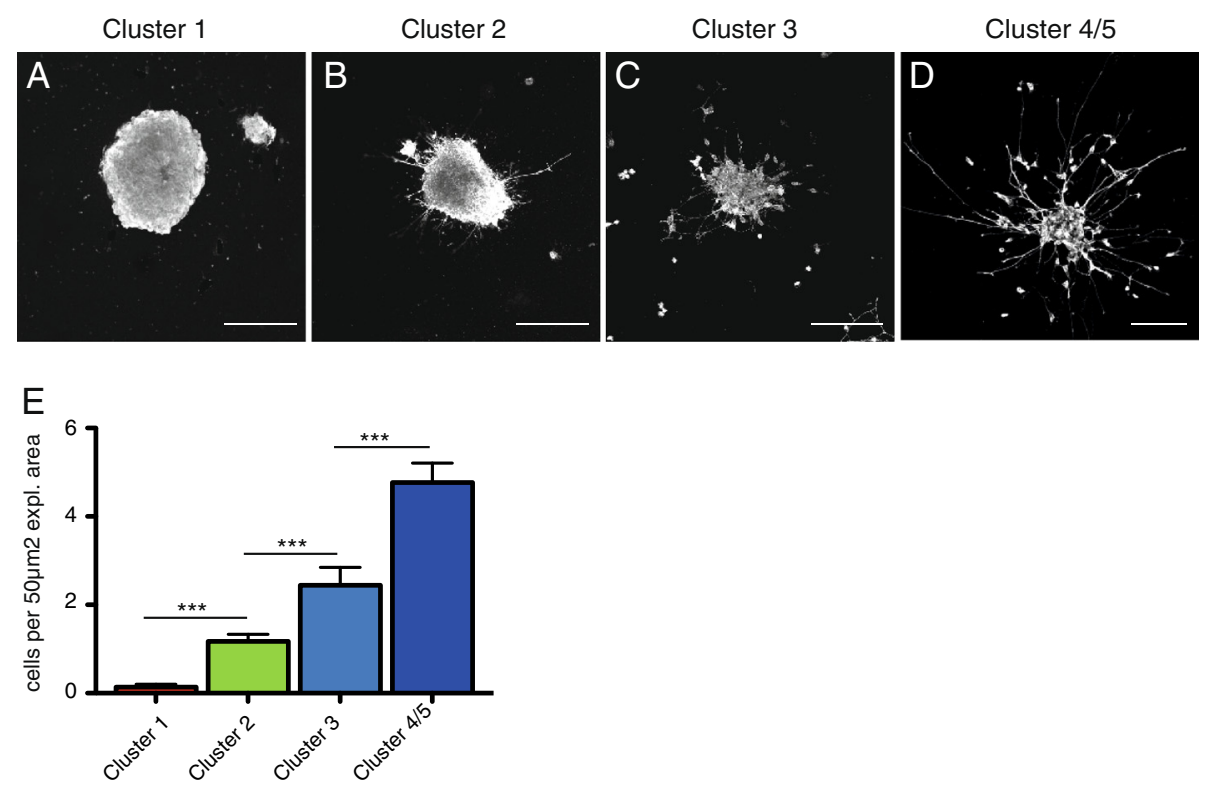

Figure 6 Migration properties. (A-D) Migration profile for cluster-specific MGE explant cultures (scale bars represent $100 \mu \mathrm{m})$. (E) Quantification of cells migrated from individual clusters per $50 \mu^{2}$ explant area (one way ANOVA analysis; ${ }^{* *} P<0.0001$, error bars represent standard error of the mean).

Seeking to close this gap is an important avenue of research. Recently, two methods based on in situ sequencing were described, allowing simultaneous detection of up to 50 RNAs [44] or whole transcriptomes [45] with subcellular resolution. However, these methods require specialized, custom-built equipment, and wholetranscriptome analysis has not yet been demonstrated on real tissue, only cultured cells. In the past, laser capture microdissection has been used to analyze defined regions, but this rests on the assumption that functionally distinct regions are known a priori. We reasoned that we could leverage the high throughput of our recently developed single-cell RNA-seq protocol, and use standard laser capture microdissection to sample a tissue in a grid at high spatial resolution, while still covering the entire transcriptome. Using a systematic sampling strategy, we obtained cubic voxels at $50 \mu \mathrm{m}$ resolution in a regular grid covering the MGE. This allowed us to project the expression of any gene onto an image representing the original tissue section. A key advantage of this method is that whole-transcriptome data are obtained from single tissue sections, in contrast to methods such as Allen Brain Atlas that use multiple sections and multiple brains for multiplexing. Our method is therefore dramatically less costly and avoids the problem of registering sections derived from different brains. Furthermore, it uses only commercially available, widely used equipment.

The information content in each image can be increased in two ways, either by increasing resolution (making the voxels smaller) or by increasing the area (cutting more voxels). Voxel size is currently limited by the capability of laser microdissection. Judging by the black laser traces in Figure $1 \mathrm{C}$, laser damage visibly affected up to $10 \mu \mathrm{m}$, and invisible damage may extend further. The surface area, on the other hand, is limited by the cost of sequencing. As these costs have dropped rapidly in recent years, our method should trivially scale to larger areas and (using adjacent sections) to three-dimensional volumetric imaging of whole-transcriptome expression data.

The sensitivity of the method is limited by losses during tissue preparation, laser microdissection and lysis/reverse transcription. Losses during tissue preparation were probably small, as we have obtained good quality RNA single-molecule fluorescence in situ hybridization results from similar sections (data not shown). The transcriptomes obtained here from $50 \mu \mathrm{m}$ voxels were of a similar depth and quality to those obtained previously, using the same methods, from approximately $15 \mu \mathrm{m}$ diameter hand-picked living cells, indicating that laser capture microdissection had caused significant losses. Optimization of the laser capture procedure, combined with recently developed more sensitive single-cell RNAseq protocols, may alleviate these concerns.

Traditionally, the delineation of progenitor regions in the mammalian forebrain has been largely based on anatomical landmarks (for example, sulci and bulges), which could be misleading since many structures undergo substantial morphological changes during development. Therefore, the identification of progenitor domains based 
on gene expression studies has become indispensable. In the MGE, previous studies identified progenitor pools within the ventricular zone based on differential expression of transcription factors, and demonstrated that the time point and birthplace of an interneuron precursor cell influence its final cell fate in the cerebral cortex [6]. We note that several genes identified in our study appear to be expressed in a gradient in the ventricular zone of the MGE, such as Sox2 and Couptf1 (Figure 4A,E). This suggests that relative quantitative differences of a few key transcription factors, rather than absolute yes/no expression, may drive the differentiation of interneuron precursors in the MGE. Such differences may be too small to translate into discrete and discontinuous functional subcompartments, like those identified here. It is interesting to note that our method was able to identify the precise boundary of the ventricular zone in an unsupervised manner, based solely on clustering of gene expression data.

No functional subdivisions of the postmitotic, mantle zone of the MGE were known to exist, and it has been largely assumed that precursor cells wander out of the ventricular zone without any particular pattern. Here, we were able to identify distinct waves of progenitor cells in the MGE mantle by means of their transcriptional pattern, which we could confirm by in situ hybridization. Based on functional annotation of the genes expressed in each cluster, we could assign functional characteristics in accordance with their anatomical localization within the MGE. We predicted a dorsoventral switch in proliferative and migratory potential, which we confirmed using functional assays. It is interesting that, although proliferation potential decreased abruptly outside the subventricular zone, migratory capacity increased more or less steadily in the MGE mantle, particularly in clusters 3 and 4/5, in agreement with the appearance of migration-related gene expression. Despite accounting for about half of the MGE mantle, very little migratory potential was detected in cells from cluster 2, consistent with an overall absence of expression of genes associated with cell migration in this cluster. It is possible, therefore, that cells from the ventricular zone enter the mantle by alternative mechanisms. We note that clusters 2, 3 and 4/5, all share high levels of neural cell adhesion molecule (NCAM) expression, which is absent in cluster 1, suggesting that postmitotic cells may extrude the ventricular zone by differential cell adhesion and only subsequently fully engage gene programs dedicated to cell migration.

\section{Conclusions}

We provide a strategy to simultaneously measure the spatial distribution of all mRNAs, which requires only commonly available equipment and reagents (laser microdissection, RNA-sequencing). The result is a 'transcriptome image, which can be mined to determine spatial domains of gene expression corresponding to functionally relevant, dynamic developmental processes.

\section{Materials and methods \\ Tissue preparation}

Wild-type and Gfra1 ${ }^{\mathrm{Tlz}}$ mutant embryos (both C57bl6/J) obtained by breeding heterozygous Gfra1-deficient mice $[13,46]$ at E12.5 were removed, immersed in Tissue Tek (Sakura, Alphen aan den Rijn, The Netherlands) and immediately snap frozen in $-80^{\circ} \mathrm{C}$ cold isopentane. Serial $50 \mu \mathrm{m}$ thick coronal sections were made using a cryostat (NX70, Thermo Scientific, Waltham, Massachusetts, USA) and collected onto frame slides pretreated according to the manufacturer's manual (MMI, Zürich, Switzerland). Sections were air dried, shortly immersed in $100 \%$ ethanol and finally transported in $50 \mathrm{ml}$ falcon tubes with desiccant on dry ice to the laser microdissection instrument. For laser microdissection, a coronal section in the middle portion of the MGE (demarked by a deep sulcus from the lateral ganglionic eminence and an obvious notch or invagination towards the pre-optic area region; Figure 1A) was chosen from each embryo. For in situ hybridization and immunohistochemistry, embryos were fixed in $4 \%$ paraformaldehyde (PFA) overnight at $4^{\circ} \mathrm{C}$ and cryoprotected by immersion in $30 \%$ sucrose. Animal protocols were approved by Stockholms Norra Djurförsöksetiska Nämnd (\#N280/20 to CFI) and are in accordance with ethical guidelines of the Karolinska Institute.

\section{Laser microdissection}

Laser microdissection was performed with a MMI Cellcut Plus instrument (MMI). Rectangles of $50 \times 50 \mu \mathrm{m}$ were cut from tissue sections at $20 \times$ magnification under bright field illumination. The following cutting parameters were used: $14 \%$ cutting velocity, $53.3 \%$ laser focus, $100 \%$ laser power, 3 cutting repeats. Focus was adapted manually while cutting. Microdissected material was collected with a sticky cap strip (MMI) using the 'cap down' mode during cutting. After isolation, $5 \mu \mathrm{l}$ cell capture mastermix was added to each sample and immediately placed on dry ice.

\section{Single-cell tagged reverse transcription}

The third version of the STRT protocol was used [11], except that the cell capture mastermix contained $1 \%$ Tween 20, $400 \mathrm{nM}$ T30 and $2 \mu \mathrm{M}$ TSO without magnesium chloride. In order to determine optimal cycle numbers, an additional quantitative PCR was performed prior to the amplification step of the original version using the following parameters: $95^{\circ} \mathrm{C}$ for 1 minute, 35 cycles of $95^{\circ} \mathrm{C}$ for $30 \mathrm{~s}, 65^{\circ} \mathrm{C}$ for $30 \mathrm{~s}$ and $68^{\circ} \mathrm{C}$ for 4 minutes followed by a final dissociation stage. The SalI digestion and ADP2 ligation were split up into two reactions. Primary data analysis was performed as previously described, and gene expression was normalized to transcripts 
per million by dividing the read counts of each gene by the total number of reads mapped to genes (exons and splice junctions), excluding repeats. The complete dataset is available through Gene Expression Omnibus [47] under accession GSE60402.

\section{Statistical analysis of transcriptome imaging}

Topological data analysis was performed using the Ayasdi software with the following parameters: Metric: norm correlation (Pearson correlation on standardized values); Lens: principal metric SVD (resolution: 40; gain: $2.5 \times$, equalized); Lens: secondary metric SVD (resolution: 30; gain: $4 \times$, equalized). Differential gene expression analysis was performed using the Kolmogorov-Smirnov non-parametric test. For functional analysis in DAVID, a threshold of a KolmogorovSmirnov score $>0.25$ was used. Detailed results of term enrichment analysis are given in Additional file 3.

\section{In situ hybridization and immunohistochemistry}

In situ hybridization and immunohistochemistry were sequentially performed on the same section. Riboprobes were derived from DNA fragments obtained by PCR from E12.5 MGE cDNA using the primers listed in Additional file 4. Riboprobe synthesis and in situ hybridization were essentially carried out as previously described [48] with a few modifications. After incubation with anti DIG antibody, sections were washed three times for 5 minutes in phosphate-buffered saline (PBS), followed by a wash in maleic acid plus Tween 20 (MABT) for 30 minutes. Following two 5 minute washes in PBS, one for 5 minutes in MABT, and one for 5 minutes in Tris buffer $(1 \mathrm{M}, \mathrm{pH}$ 9.5), fluorescent staining was developed overnight at room temperature using Fast Red solution (Sigma, St. Louis, Missouri, USA). After washing in PBS $(3 \times 5$ minutes $)$ sections were blocked (5\% serum in PBS plus $0.3 \%$ TritonX) for an hour at room temperature before incubation overnight in primary antibody (rat anti-BrdU (1:500; \#YSRTMCA2060GA, Accurate Chemicals, Westbury, New York, USA) and rabbit anti-GAP43 (1:500; NB300-143, Chemicon, Billerica, Massachusetts, USA)). For BrdU staining, denaturation of DNA was achieved by incubation in $1 \mathrm{M}$ $\mathrm{HCl}$ for 45 minutes at $45^{\circ} \mathrm{C}$ prior to blocking. Sections were developed by incubation in secondary antibody solution (Alexa 488 and 645, Invitrogen, Carlsbad, California, USA) before finally being cover slipped in Fluorescent Mounting Medium (DAKO, Carpinteria, California, USA). In situ hybridizations on tissue samples were repeated three to five times including a sense control for each individual riboprobe.

\section{BrdU staining}

Time pregnant (E12.5) wild-type females were intraperitoneally injected with one dose of BrdU $(100 \mathrm{mg} / \mathrm{kg}$, Roche, Basel, Switzerland) and sacrificed 30 minutes after injection by cervical dislocation. Embryos were removed, fixed in 4\% PFA and processed for in situ hybridization as above. BrdU-labeled cells from six MGE sections per embryo (wild type, $\mathrm{N}=3$ ) were counted and the data were subjected to one-way ANOVA analysis (Prism v5, Graphpad, La Jolla, California, USA).

\section{Migration assay}

Embryos (E12.5) were collected and embedded in 5\% low melting agarose (Sigma). Coronal sections (100 $\mu \mathrm{m}$ thick) were cut using a vibratome (Leica VT1000, Wetzlar, Germany). Sections were transferred onto polylysin coated coverslips. The MGE was separated from each brain section and subsequently split into small pieces using two needles. The tissue fragments were embedded in Matrigel (growth factor reduced, BD, Franklin Lakes, New Jersey, USA) and cultured in neurobasal medium (Gibco, Carlsbad, California, USA) supplemented with $2 \%$ B27, glucose, $200 \mathrm{mM}$ glutamine and antibiotics for $24 \mathrm{~h}$. After $20 \mathrm{~h}$ in culture, BrdU (10 $\mu \mathrm{M}$; Roche) was added to the medium. Tissue areas were fixed in 4\% PFA and immunostained as described above. Cell migration was assessed by counting neurons that had migrated out of the tissue explant normalized to $50 \mu \mathrm{m}^{2}$ explant area. Fifteen to 24 explants were used for each cluster from three individual litters. Statistical analysis was performed by one-way ANOVA (Prism v5).

\section{Additional files}

Additional file 1: Topographical expression map for additional animals. Topographical expression map within the MGE of the same genes shown in Figure 1D (expression level given in reads per million). (A) Wild-type animal 2; (B) Gfra ${ }^{\text {tz/tlz }}$ animal.

Additional file 2: Showing validation of the dissection method by in situ hybridization against cluster-specific markers (first column) combined with Gap43 (second column) and BrdU (third column) staining (merge shown in column 4; scale bars represent $100 \mu \mathrm{m}$ ).

Additional file 3: Providing detailed results of Gene Ontology analysis, including results of statistical gene enrichment tests.

Additional file 4: Showing primer sequences used for riboprobe synthesis.

\section{Abbreviations}

BrdU: bromodeoxyuridine; E: embryonic day; GO: Gene Ontology; MGE: medial ganglionic eminence; PBS: phosphate-buffered saline; PCR: polymerase chain reaction; PFA: paraformaldehyde; RNA-seq: RNA-sequencing; STRT: single-cell tagged reverse transcription; TDA: topological data analysis.

\section{Competing interests}

The authors declare that they have no competing interests.

\section{Authors' contributions}

SL and CFI conceived the study and planned experiments. SZ prepared tissues, performed laser capture microdissection, performed proliferation and migration assays and analyzed data. PZ performed laser capture microdissection and RNA-seq, and analyzed data. PL wrote the bioinformatics pipeline, analyzed data and evaluated clustering methods. All authors wrote and revised the manuscript. All authors read and approved the final manuscript. 


\section{Acknowledgements}

We thank Tanya Petrossian (Ayasdi Inc.) and Damir Herman (Ayasdi, Inc.) for helpful discussions on topological data analysis; Ayasdi, Inc. for use of the Iris software package; and Anna Johnsson for performing DNA sequencing. This work was supported by grants from the Swedish Research Council, the VIIth Framework Programme of the European Union ('Molpark' network) to CFI and SZ, the Linneaus Center in Developmental Biology and Strategic Research Program in Regenerative Medicine to $\mathrm{CFI}$, a Post-PhD research grant from Wenner Gren Foundation to SZ, European Research Council Starting Grant 261063 to SL and Swedish Foundation for Strategic Research grant MDB09 to SL.

\section{Author details}

${ }^{1}$ Department of Neuroscience, Karolinska Institute, Stockholm SE-171 77, Sweden. ${ }^{2}$ Department of Medical Biochemistry and Biophysics, Karolinska Institute, Stockholm SE-171 65, Sweden. ${ }^{3}$ Life Sciences Institute, Department of Physiology, National University of Singapore, Singapore 117456, Singapore.

Received: 16 April 2014 Accepted: 3 October 2014

Published online: 25 October 2014

\section{References}

1. Petilla Interneuron Nomenclature Group, Ascoli GA, Alonso-Nanclares L, Anderson SA, Barrionuevo G, Benavides-Piccione R, Burkhalter A, Buzsáki G, Cauli B, Defelipe J, Fairén A, Feldmeyer D, Fishell G, Fregnac Y, Freund TF, Gardner D, Gardner EP, Goldberg JH, Helmstaedter M, Hestrin S, Karube F, Kisvárday ZF, Lambolez B, Lewis DA, Marín O, Markram H, Muñoz A, Packer A, Petersen $C C H$, Rockland KS, et al: Petilla terminology: nomenclature of features of GABAergic interneurons of the cerebral cortex. Nat Rev Neurosci 2008, 9:557-568

2. Defelipe J, López-Cruz PL, Benavides-Piccione R, Bielza C, Larrañaga P, Anderson S, Burkhalter A, Cauli B, Fairén A, Feldmeyer D, Fishell G, Fitzpatrick D, Freund TF, González-Burgos G, Hestrin S, Hill S, Hof PR, Huang J, Jones EG, Kawaguchi Y, Kisvárday Z, Kubota Y, Lewis DA, Marín O, Markram H, McBain CJ, Meyer HS, Monyer H, Nelson SB, Rockland K, et al: New insights into the classification and nomenclature of cortical GABAergic interneurons. Nat Rev Neurosci 2013, 14:202-216.

3. Inan M, Welagen J, Anderson SA: Spatial and temporal bias in the mitotic origins of somatostatin- and parvalbumin-expressing interneuron subgroups and the chandelier subtype in the medial ganglionic eminence. Cereb Cortex 2012, 22:820-827.

4. Fogarty M, Grist M, Gelman D, Marín O, Pachnis V, Kessaris N: Spatial genetic patterning of the embryonic neuroepithelium generates GABAergic interneuron diversity in the adult cortex. J Neurosci 2007 27:10935-10946.

5. Xu Q, Cobos I, La Cruz DE, Rubenstein JL, Anderson SA: Origins of cortical interneuron subtypes. J Neurosci 2004, 24:2612-2622.

6. Flames N, Pla R, Gelman DM, Rubenstein JLR, Puelles L, Marín O: Delineation of multiple subpallial progenitor domains by the combinatorial expression of transcriptional codes. J Neurosci 2007 27:9682-9695.

7. Miyoshi G, Fishell G: GABAergic interneuron lineages selectively sort into specific cortical layers during early postnatal development. Cereb Cortex 2011, 21:845-852.

8. Lein ES, Hawrylycz MJ, Ao N, Ayres M, Bensinger A, Bernard A, Boe AF, Boguski MS, Brockway KS, Byrnes EJ, Chen L, Chen L, Chen T-M, Chin MC, Chong J, Crook BE, Czaplinska A, Dang CN, Datta S, Dee NR, Desaki AL, Desta T, Diep E, Dolbeare TA, Donelan MJ, Dong H-W, Dougherty JG, Duncan BJ, Ebbert AJ, Eichele G, et al: Genome-wide atlas of gene expression in the adult mouse brain. Nature 2007, 445:168-176.

9. Larsson C, Grundberg I, Söderberg O, Nilsson M: In situ detection and genotyping of individual mRNA molecules. Nat Methods 2010, 7:395-397.

10. Islam S, Kjällquist U, Moliner A, Zajac P, Fan J-B, Lönnerberg P, Linnarsson S: Characterization of the single-cell transcriptional landscape by highly multiplex RNA-seq. Genome Res 2011, 21:1160-1167.

11. Islam S, Llquist UKA, Moliner A, Zajac P, Fan J-B, Nnerberg PLO, Linnarsson S: Highly multiplexed and strand-specific single-cell RNA 5 ' end sequencing. Nat Protoc 2012, 7:813-828.

12. Enomoto H, Araki T, Jackman A, Heuckeroth RO, Snider WD, Johnson EM, Milbrandt J: GFR alpha1-deficient mice have deficits in the enteric nervous system and kidneys. Neuron 1998, 21:317-324.
13. Canty AJ, Dietze J, Harvey M, Enomoto H, Milbrandt J, Ibáñez CF: Regionalized loss of parvalbumin interneurons in the cerebral cortex of mice with deficits in GFRalpha1 signaling. J Neurosci 2009, 29:10695-10705.

14. Pozas E, Ibáñez CF: GDNF and GFRalpha1 promote differentiation and tangential migration of cortical GABAergic neurons. Neuron 2005, 45:701-713

15. O'Donoghue SI, Gavin A-C, Gehlenborg N, Goodsell DS, Hériché J-K, Nielsen CB, North C, Olson AJ, Procter JB, Shattuck DW, Walter T, Wong B: Visualizing biological data-now and in the future. Nat Methods 2010, 7:S2-S4.

16. Lum PY, Singh G, Lehman A, Ishkanov T, Vejdemo-Johansson M, Alagappan M, Carlsson J, Carlsson G: Extracting insights from the shape of complex data using topology. Sci Rep 2013, 3:1236.

17. Huang DW, Sherman BT, Lempicki RA: Bioinformatics enrichment tools: paths toward the comprehensive functional analysis of large gene lists. Nucleic Acids Res 2009, 37:1-13.

18. Huang DW, Sherman BT, Lempicki RA: Systematic and integrative analysis of large gene lists using DAVID bioinformatics resources. Nat Protoc 2009, 4:44-57.

19. Aoki I, Higuchi M, Gotoh Y: NEDDylation controls the target specificity of E2F1 and apoptosis induction. Oncogene 2013, 32:3954-3964.

20. Palm T, Hemmer K, Winter J, Fricke IB, Tarbashevich K, Sadeghi Shakib F, Rudolph I-M, Hillje A-L, De Luca P, Bahnassawy L, Madel R, Viel T, De Siervi A, Jacobs AH, Diederichs S, Schwamborn JC: A systemic transcriptome analysis reveals the regulation of neural stem cell maintenance by an E2F1-miRNA feedback loop. Nucleic Acids Res 2013, 41:3699-3712.

21. Nobs L, Nestel S, Kulik A, Nitsch C, Atanasoski S: Cyclin D1 is required for proliferation of Olig2-expressing progenitor cells in the injured cerebral cortex. Glia 2013, 61:1443-1455.

22. Colasante $G$, Collombat P, Raimondi V, Bonanomi D, Ferrai C, Maira M, Yoshikawa K, Mansouri A, Valtorta F, Rubenstein JLR, Broccoli V: Arx is a direct target of DIx2 and thereby contributes to the tangential migration of GABAergic interneurons. J Neurosci 2008, 28:10674-10686.

23. Flandin $P$, Zhao $Y$, Vogt $D$, Jeong J, Long J, Potter $G$, Westphal $H$, Rubenstein JLR: Lhx6 and Lhx8 coordinately induce neuronal expression of Shh that controls the generation of interneuron progenitors. Neuron 2011, 70:939-950

24. Du T, Xu Q, Ocbina PJ, Anderson SA: NKX2.1 specifies cortical interneuron fate by activating Lhx6. Development 2008, 135:1559-1567.

25. Wang Y, Dye CA, Sohal V, Long JE, Estrada RC, Roztocil T, Lufkin T, Deisseroth K, Baraban SC, Rubenstein JLR: Dlx5 and Dlx6 regulate the development of parvalbumin-expressing cortical interneurons. J Neurosci 2010, 30:5334-5345.

26. Long JE, Cobos I, Potter GB, Rubenstein JLR: DIx1\&2 and Mash1 transcription factors control MGE and CGE patterning and differentiation through parallel and overlapping pathways. Cereb Cortex 2009, 19:i96-i106.

27. Graham V, Khudyakov J, Ellis P, Pevny L: SOX2 functions to maintain neural progenitor identity. Neuron 2003, 39:749-765.

28. Zappone MV, Galli R, Catena R, Meani N, De Biasi S, Mattei E, Tiveron C, Vescovi AL, Lovell-Badge R, Ottolenghi S, Nicolis SK: Sox2 regulatory sequences direct expression of a (beta)-geo transgene to telencephalic neural stem cells and precursors of the mouse embryo, revealing regionalization of gene expression in CNS stem cells. Development 2000, 127:2367-2382.

29. Li C, Ito H, Fujita K, Shiwaku H, Qi Y, Tagawa K, Tamura T, Okazawa H: Sox2 transcriptionally regulates PQBP1, an intellectual disability-microcephaly causative gene, in neural stem progenitor cells. PLoS One 2013, 8:e68627.

30. Long JE, Swan C, Liang WS, Cobos I, Potter GB, Rubenstein JLR: DIx1\&2 and Mash1 transcription factors control striatal patterning and differentiation through parallel and overlapping pathways. J Comp Neurol 2009, 512:556-572.

31. Kerjan G, Koizumi H, Han EB, Dubé CM, Djakovic SN, Patrick GN, Baram TZ, Heinemann SF, Gleeson JG: Mice lacking doublecortin and doublecortin-like kinase 2 display altered hippocampal neuronal maturation and spontaneous seizures. Proc Natl Acad Sci U S A 2009, 106:6766-6771.

32. Wang $Y$, Lin L, Lai $H$, Parada LF, Lei L: Transcription factor Sox 11 is essential for both embryonic and adult neurogenesis. Dev Dyn 2013, 242:638-653.

33. Mouri A, Sasaki A, Watanabe K, Sogawa C, Kitayama S, Mamiya T, Miyamoto Y, Yamada K, Noda Y, Nabeshima T: MAGE-D1 regulates expression of depression-like behavior through serotonin transporter ubiquitylation. J Neurosci 2012, 32:4562-4580. 
34. Cobos I, Borello U, Rubenstein JLR: Dlx transcription factors promote migration through repression of axon and dendrite growth. Neuron 2007, 54:873-888.

35. Ryu S, McDonnell K, Choi H, Gao D, Hahn M, Joshi N, Park S-M, Catena R, Do Y, Brazin J, Vahdat LT, Silver RB, Mittal V: Suppression of miRNA-708 by polycomb group promotes metastases by calcium-induced cell migration. Cancer Cell 2013, 23:63-76.

36. Eißßmann M, Gutschner T, Hämmerle M, Günther S, Caudron-Herger M, Groß M, Schirmacher P, Rippe K, Braun T, Zörnig M, Diederichs S: Loss of the abundant nuclear non-coding RNA MALAT1 is compatible with life and development. RNA Biol 2012, 9:1076-1087.

37. Pilz G-A, Shitamukai A, Reillo I, Pacary E, Schwausch J, Stahl R, Ninkovic J, Snippert HJ, Clevers H, Godinho L, Guillemot F, Borrell V, Matsuzaki F, Götz M: Amplification of progenitors in the mammalian telencephalon includes a new radial glial cell type. Nat Commun 2013, 4:2125.

38. Glickstein SB, Alexander S, Ross ME: Differences in cyclin D2 and D1 protein expression distinguish forebrain progenitor subsets. Cereb Cortex 2007, 17:632-642.

39. Glickstein SB, Moore H, Slowinska B, Racchumi J, Suh M, Chuhma N, Ross ME: Selective cortical interneuron and GABA deficits in cyclin D2-null mice. Development 2007, 134:4083-4093.

40. Lyubimova A, Itzkovitz S, Junker JP, Fan ZP, Wu X, van Oudenaarden A: Single-molecule mRNA detection and counting in mammalian tissue. Nat Protoc 2013, 8:1743-1758.

41. Rust MJ, Bates M, Zhuang X: Sub-diffraction-limit imaging by stochastic optical reconstruction microscopy (STORM). Nat Methods 2006, 3:793-795.

42. Betzig E, Patterson GH, Sougrat R, Lindwasser OW, Olenych S, Bonifacino JS, Davidson MW, Lippincott-Schwartz J, Hess HF: Imaging intracellular fluorescent proteins at nanometer resolution. Science 2006, 313:1642-1645.

43. Tang F, Barbacioru C, Wang Y, Nordman E, Lee C, Xu N, Wang X, Bodeau J, Tuch BB, Siddiqui A, Lao K, Surani MA: mRNA-Seq whole-transcriptome analysis of a single cell. Nat Methods 2009, 6:377-382.

44. Ke R, Mignardi M, Pacureanu A, Svedlund J, Botling J, Wählby C, Nilsson M: In situ sequencing for RNA analysis in preserved tissue and cells. Nat Methods 2013, 10:857-860.

45. Lee JH, Daugharthy ER, Scheiman J, Kalhor R, Yang JL, Ferrante TC, Terry R, Jeanty SSF, Li C, Amamoto R, Peters DT, Turczyk BM, Marblestone AH, Inverso SA, Bernard A, Mali P, Rios X, Aach J, Church GM: Highly multiplexed subcellular RNA sequencing in situ. Science 2014, 343:1360-1363.

46. Enomoto H, Hughes I, Golden J, Baloh RH, Yonemura S, Heuckeroth RO, Johnson EM, Milbrandt J: GFRalpha1 expression in cells lacking RET is dispensable for organogenesis and nerve regeneration. Neuron 2004, 44:623-636.

47. Edgar R, Domrachev M, Lash AE: Gene Expression Omnibus: NCBI gene expression and hybridization array data repository. Nucleic Acids Res 2002, 30:207-210

48. Zechel S, Huber-Wittmer K, von Bohlen, Halbach O: Distribution of the iron-regulating protein hepcidin in the murine central nervous system. J Neurosci Res 2006, 84:790-800.

doi:10.1186/s13059-014-0486-z

Cite this article as: Zechel et al:: Topographical transcriptome mapping of the mouse medial ganglionic eminence by spatially resolved RNA-seq. Genome Biology 2014 15:486.

\section{Submit your next manuscript to BioMed Central and take full advantage of:}

- Convenient online submission

- Thorough peer review

- No space constraints or color figure charges

- Immediate publication on acceptance

- Inclusion in PubMed, CAS, Scopus and Google Scholar

- Research which is freely available for redistribution

Submit your manuscript at www.biomedcentral.com/submit
C BiolMed Central 